\title{
Indirect interaction of impurity spins on the surface of topological insulators
}

\author{
A. V. Ten ${ }^{1}$, M. B. Belonenko ${ }^{2}$ \\ ${ }^{1}$ Volgograd State University, Volgograd, Russia \\ ${ }^{2}$ Volgograd Institute of Business, Volgograd, Russia \\ ten.anastasia@volsu.ru,mbelonenko@yandex.ru
}

PACS 75.75 -c, 73.20 Hb, 73.22 Pr

DOI 10.17586/2220-8054-2017-8-3-386-390

\begin{abstract}
In this work, a mathematical model to study the indirect interaction in topological insulators was constructed. Analysis of the model was carried out numerically. We have calculated the indirect exchange interaction in the film of a topological insulator, for example $\mathrm{Bi}_{2} \mathrm{Te}_{3}$, within the $\mathrm{s}-\mathrm{d}$ model. The calculations showed that the magnetic ordering of the impurity spins varies periodically with increasing distance between atoms, asymptotically decreasing to zero. $\lambda$ is a parameter associated with hexagonal distortion and is a component of the dispersion relation. The dependence of the constants of the effective exchange interaction upon the $\lambda$ parameter is shown; this parameter characterizes the crystal lattice geometry for a topological insulator.
\end{abstract}

Keywords: topological insulators, impurity spin, indirect exchange, s-d model.

Received: 23 January 2017

Revised: 28 February 2017

Final revision: 26 May 2017

\section{Introduction}

Currently, scientists are increasingly interested in the study of topological insulators [1], and questions about the interaction of topological insulators with different kinds of impurities that could exist in a thin film of this material.

Different applications perspective of spintronics, based, in particular, on the effects of spin-spin interactions, has been confirmed theoretically and experimentally [2-4]. This focused on the main mechanism of the interaction of spins because it is an indirect mechanism (e.g. RKKY, through the conduction electrons)) in the cases of such potentially useful substances such as graphene, bigraphene, systems of quantum dots, in article [5-7].

All of the above makes the task of consideration of collective effects related to the interaction of impurities with the electrons of a thin film of a topological insulator, i.e., indirect exchange interaction [8,9], highly relevant.

Particularly strong "spin-orbit coupling" (tight coupling between the spin orientation and the direction of its movement) in the atoms of heavy elements such as bismuth may generate topological invariants that lead to the most radical consequences for the material properties. In fact, inside the semiconductor and the insulator becomes "metallic" on the surface of the conductor. At the same time, due to the spin-orbit interaction and its consequences, the material properties are very attractive from the point of view of practical applications. Researchers have long been trying to create a radically new direction in electronics - spintronic devices that are optimal for the storage and transmission of information would use the spin of electrons rather than their displacement. The fragile nature of quantum effects is difficult to manage and is very strongly inhibits the development of practical devices, well, in fact the nature of the phenomenon of spin TI promises prospects for progress.

\section{Basic equations}

We consider a film of a topological insulator within the framework of the model described in [10].

We will choose the Hamiltonian system as the standard form for the $\mathrm{s}-\mathrm{d}$ exchange models [11]:

$$
\begin{gathered}
H=H_{0}+H_{i n t}, \\
H_{0}=\sum_{k, \sigma} \varepsilon_{k} a_{k \sigma}^{+} a_{k \sigma}, \quad H_{i n t}=\sum_{n n^{\prime}} J(\mathbf{q}) \sum_{\sigma \sigma^{\prime}} S_{q} \sigma_{\sigma \sigma^{\prime}} a_{n \sigma}^{+} a_{n^{\prime} \sigma^{\prime}},
\end{gathered}
$$

where $S_{q}=\sum_{R} S_{R} \exp (i \mathbf{q R}) ; \mathbf{q}=\mathbf{n}-\mathbf{n}^{\prime} ; J(\mathbf{q})-$ Fourier transform of interaction potential d-impurities with the electrons in the conduction band; $\sigma_{\sigma \sigma \prime}$ - the Pauli matrices; $S_{R}$ - is the spin vector of the impurity, located at the point R; $a_{k \sigma}^{+}, a_{k \sigma}$ - the creation operators and annihilation of electrons with spins $\sigma$ and with wave vectors $k$. 
Next, we considered a thin film of a topological insulator, we chose the Hamiltonian for this in the long-wave approximation [12]:

$$
\begin{aligned}
& H_{0}=\left(p_{x}^{2}+p_{y}^{2}\right) / 2 m+v_{F}\left(p_{x} \sigma_{y}-p_{y} \sigma_{x}\right)+\frac{\lambda}{2}\left(p_{+}^{3}+p_{-}^{3}\right) \\
& p_{ \pm}=p_{x} \pm i p_{y} .
\end{aligned}
$$

The derivation of the effective Hamiltonian (2) for a thin film starting from the Hamiltonian for a bulk sample is presented in a number of papers, of which, we only mention [14].

In expression (2), $p_{x}, p_{y}$ - components of the electron momentum, $m$ is the effective electron mass, $v_{F}-$ the Fermi velocity, $\lambda$ is a parameter associated with hexagonal distortion and is a component of the dispersion relation. Typical values of the Hamiltonian parameters, for example for $\mathrm{Bi}_{2} \mathrm{Te}_{3}$, is [13]: $m \propto 35 \mathrm{eV}^{-1} \AA^{-2}$, $v_{f} \propto 5 \cdot 10^{-4} \mathrm{eV}^{-1} \AA$.

The Hamiltonian (2) is easily diagonalized and sets the range of the electrons:

$$
\varepsilon\left(p_{x}, p_{y}\right)=\left(p_{x}^{2}+p_{y}^{2}\right) / 2 m+\sqrt{v_{f}^{2}\left(p_{x}^{2}+p_{y}^{2}\right)+\frac{\lambda^{2} p_{x}}{2}\left(p_{x}^{2}-3 p_{y}^{2}\right)} .
$$

Thus, expression (2) can be inserted in (1).

The Frohlich method of calculating indirect interactions [14] is based on the assumption that for the operators of the matrix elements, the following inequality $\left|H_{\text {int }}\right| \ll\left|H_{0}\right|$ holds, and consists of two stages. The first is the transition from the form (1) to a new form with the help of the unitary transformation, $U=\exp (-L)$, where $L-$ is anti-Hermitian operator satisfying the condition:

$$
H_{\text {int }}+\left[H_{0}, L\right]=0
$$

In the new view, the Hamiltonian $\mathrm{H}$ takes the form of:

$$
H \rightarrow \tilde{H}=H_{0}+\frac{1}{2}\left[H_{\text {int }}, L\right]+O\left(H_{\text {int }}^{3}\right) .
$$

A solution of the operator equation (4) will take the following expression [13]:

$$
L=\frac{1}{i \hbar} \lim _{\varepsilon \rightarrow 0} \int_{-\infty}^{0} e^{\varepsilon t} H_{i n t}(t) d t, \quad H_{\text {int }}(t)=\exp \left(\frac{i H_{0} t}{\hbar}\right) H_{i n t} \exp \left(-\frac{i H_{0} t}{\hbar}\right) .
$$

The second stage consists of averaging the expression (4) for the transformed Hamiltonian $\tilde{H}$ on the condition of the interactions of the field-vector: in averaging with the equilibrium density matrix for the electronic subsystem. Thus, the 2-nd order term in perturbation theory $H_{s s}=\frac{1}{2}\left\langle\left[H_{i n t}, L\right]\right\rangle$, where angular brackets denote the average value with the equilibrium density matrix for the electronic subsystems in the expansion (5) cease depending on the creation operators and annihilation of electrons, but maintains the dependence on the spin operators of different impurity atoms and, therefore, has meaning for the indirect interaction operator.

After calculation of the respective switches, the operator $L$ has the form:

$$
L=\sum_{p p^{\prime}} J(\mathbf{q}) \sum_{\sigma}\left\{\frac{\left(S_{q}^{x}-i S_{q}^{y}\right) a_{p, \sigma}^{+} a_{p^{\prime},-\sigma}}{\varepsilon_{p^{\prime}}-\varepsilon_{p}+\hbar \omega_{0}}+\frac{\left(S_{q}^{x}+i S_{q}^{y}\right) a_{p,-\sigma}^{+} a_{p^{\prime}, \sigma}}{\varepsilon_{p^{\prime}}-\varepsilon_{p}-\hbar \omega_{0}}\right\} .
$$

Substituting (6) into (5) and thermo dynamical averaging gives:

$$
H_{s s}=2 \sum_{p_{1} p_{2} R_{1} R_{2}} M\left(\exp \left(i\left(p_{1}-p_{2}\right)\left(R_{1}-R_{2}\right)\right) S_{R_{1}}^{-} S_{R_{2}}^{-}+\exp \left(i\left(p_{2}-p_{1}\right)\left(R_{1}-R_{2}\right)\right) S_{R_{1}}^{-} S_{R_{2}}^{+}\right) .
$$

Where, we have introduced the following notation for constants of the exchange interaction:

$$
M=\frac{1}{2} J_{p_{1}-p_{2}} J_{p_{2}-p_{1}}\left\{\frac{\exp \left(-\beta \varepsilon_{p_{1}}\right)-\exp \left(-\beta \varepsilon_{p_{2}}\right)}{\varepsilon_{p_{1}}-\varepsilon_{p_{2}}}\right\},
$$

where $M$ is the meaningful constants indirect exchange interaction of the impurities' spins, carried out by crystallite conduction electrons.

We have considered the exchange interaction of localized impurities of the spins in the direct space; for that, we have applied the inverse Fourier transform (in reciprocal space, integration was carried out at the Brillouin zone):

$$
J_{e f f}=\int_{0}^{\frac{\pi}{3 a}} d k_{x 1} \int_{0}^{\frac{\sqrt{3}}{3} k_{x 1}} d k_{y 1} \int_{0}^{\frac{\pi}{3 a}} d k_{x 2} \int_{0}^{\frac{\sqrt{3}}{3} k_{x 2}} 2 M\left(k_{x 1}, k_{y 1}, k_{x 2}, k_{y 2}\right) \exp \left\{i\left(k_{x 1}-k_{x 2}\right) x\right\} \exp \left\{i\left(k_{y 1}-k_{y 2}\right) y\right\} d k_{y 2} .
$$


We would like to note that the oscillating part is already contained implicitly in this dependence and by evaluating the integrals with the pass method, it is possible to show the existence of exponential decline, i.e. strong magnitude reduce of indirect interactions with increasing distances between the impurities. We would like to point out that this behavior is always typical for mechanism of indirect exchange through the conduction electrons and caused by the local character of the interaction of electrons with impurity atoms.

Exchange interaction depends on the distance and the direction, but we consider only the dependence on the distance. The dependence of the exchange interaction on the direction is in the Hamiltonian for electrons. This is lost after averaging over the electronic subsystem states.

\section{The results of numerical simulation}

Due to a rather complicated dependence of the resulting operator of the indirect interaction from the task parameters, the found parameters were analyzed numerically. Thus, Fig. 1 shows a typical dependence of constants of the interaction exchange (having the meaning of system energy, depending only on the impurity spin orientation) between the components of the impurity spin from the distance for different values of parameter $\lambda$. This parameter characterizes the complexity of the studied system's geometry in contrast to the usual square lattice.

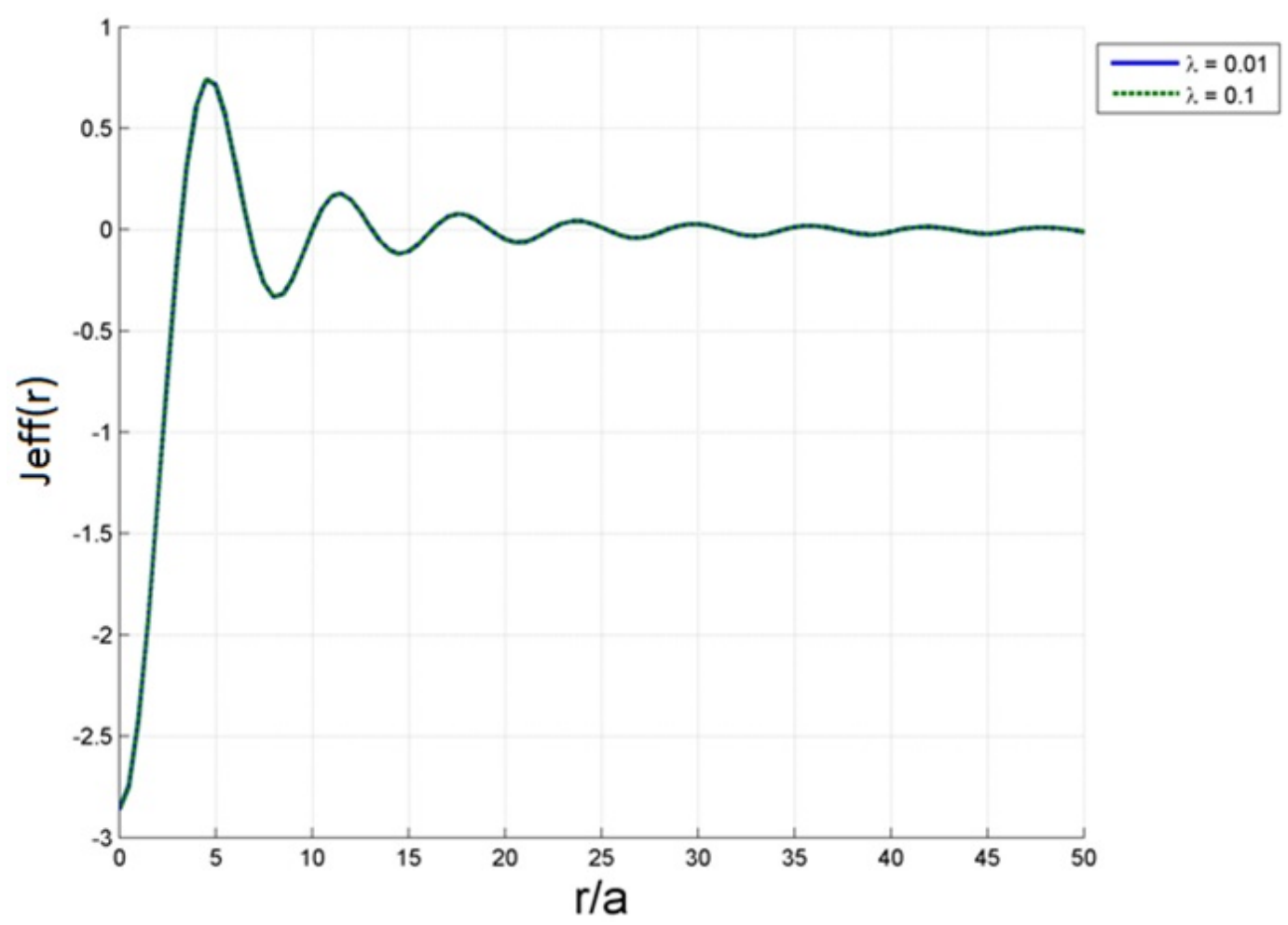

FIG. 1. The dependence of the indirect interaction constants on the distance for small $\lambda$. The distance between the localized spins is expressed in units of the lattice constant $a=2.49 \AA$

Figures 1 and 2 show the dependence of the exchange interaction constants on the parameter $\lambda$. As can be seen from the figures, the value of the exchange interaction constant is greater than in the case of large values of the parameter $\lambda$.

The obtained results confirm the possibility of formation of areas with ferromagnetic or antiferromagnetic type of ordering (change of the sign of the exchange interaction constant) the impurity atoms with variation geometry of the lattice, which is undoubtedly important for different applications.

\section{Conclusion}

The peculiarity of the indirect interaction of the electron spins with the help of topological insulator films studied in this work is that the exchange interaction was considered, based on the long-wave approximation. This dispersion relation includes pulses of electrons near the Dirac points.

In conclusion, we will formulate the main inference of this work: 


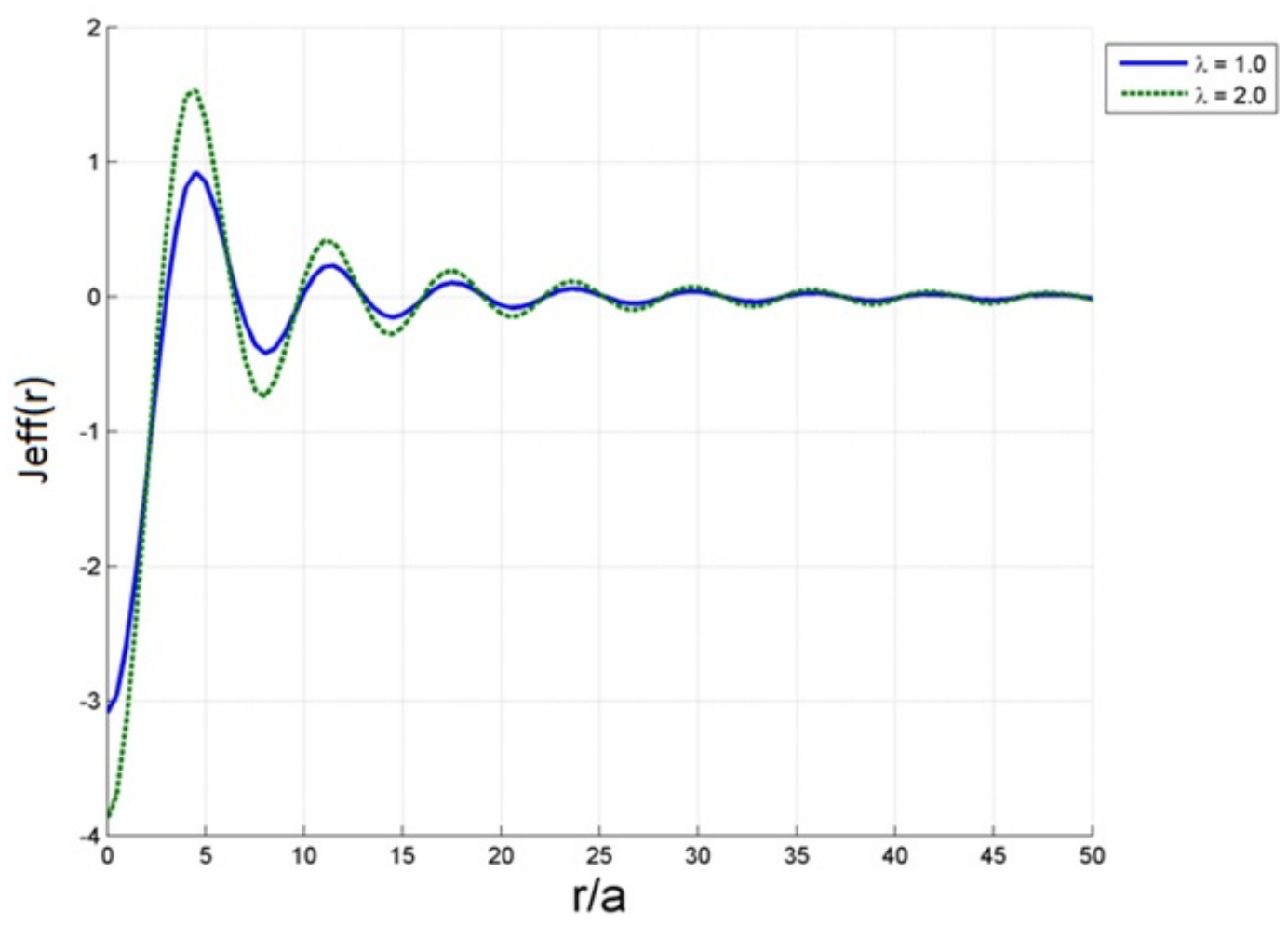

FIG. 2. The dependence of the constants of the indirect interaction on the distance for bigger $\lambda$. The distance between the localized spins is expressed in units of the lattice constant $a=2.49 \AA$

1. The increase of the constant $\lambda$ leads to an increase in the constants' amplitude of the indirect interaction of the impurities, depending on the distance that could be associated with the configuration change of the crystal lattice

2. The General nature of the indirect interaction has a form which is similar to other systems, the periodic dependence of the exchange interaction constants on the distance between the impurity spins, which decreases to zero asymptotically with increasing distance.

We use a monograph White "Quantum theory of magnetism" to compare our results with the results obtained in classical metallic systems. The spins of the conduction electrons created the oscillating polarization in classical metallic systems with the introduction into the metal localized moment. Spin density oscillations have the same shape as the Friedel charge density oscillations that occur, screening the charge impurity by electron gas. Indirect interaction in topological insulators and metal systems is similar, but the decrease of the interaction value of the distance in our study system is much faster. This fact, in our opinion, is due to the presence of a complex spin-orbit interaction in topological insulators.

\section{Acknowledgments}

This work was supported by Russian Foundation for Basic Research (grants No. 16-32-00230 mol a, No. 1607-01265), mathematical and numerical modeling was carried out within the framework of government task by the Ministry of Education and Science of the Russian Federation (research work title No. 2.852.2017/4.6). 


\section{References}

[1] Lin H., Markiewicz R.S., Wray L.A., Fu L., Hasan M.Z., Bansil A. Single-Dirac-Cone Topological Surface States in the T1BiSe 2 Class of Topological Semiconductors. Phys. Rev Lett., 2010, 105, P. 036404.

[2] Fert A. The origin, development and future of spintronics. Physics-Uspekhi, 2008, 178, P. 1336.

[3] Zutic I., Fabian J., Das Sarma S. Spintronics: Fundamentals and applications. Rev. Mod. Phys., 2004, 76, P. 323.

[4] Gulyaev Yu.V., Zilbelman P.E., Panas A.I., Epstein E.M. Spintronics: exchange switching of ferromagnetic metallic junctions at a low current density. Physics-Uspekhi, 2009, 179, P. 359.

[5] Belonenko M.B., Lebedev N.G., Pak A.V. Features of the indirect interaction in the doped bilayer graphene within the s-d-model. Physics of the Solid State, 2011, 53(8), P. 1604.

[6] Belonenko M.B., Lebedev N.G., Pak A.V. Study indirect interaction in bilayer graphene quantum dots within the s-d-model, Applied Physics Letters, 2011, 37(15), P. 69.

[7] Zhukov A.V., Bouffanais R., Pak A.V., Belonenko M.B. Study of the indirect exchange interaction in a strained graphene nanoribbon. Physica B, 2013, 419, P. 62-65.

[8] Dugaev V.K., Litvinov V.I., Barnas J. Phys. Rev. B, Exchange interaction of magnetic impurities in graphene. 2006, 74, P. 224438.

[9] Saeed Saremi. RKKY in half-filled bipartite lattices: Graphene as an example. Phys. Rev. B, 2007, 76, P. 184430.

[10] Konobeeva N.N., Belonenko M.B., Zhukov A.V. Few-Cycle Optical Pulses in Thin Film of Topological Insulator with Taking into Account the Nonlinearity of Medium. Universal Journal of Physics and Application, 2013, 1(3), P. 232-235.

[11] White R. Quantum theory of magnetism. Mir, M. 1988, 304 pp.

[12] Lin H., Markiewicz R.S., Wray L.A., Fu L., Hasan M.Z., Bansil A. Physical Review Letter. Single-Dirac-Cone Topological Surface States in the TlBiSe 2 . Class of Topological Semiconductors, 2010, 105, P. 036404.

[13] Reinthaler R.W., Hankiewicz E.M. Interplay of bulk and edge states in transport of two-dimensional topological insulators. Phys. Rev. B, 2012, 85, P. 165450.

[14] Xing Ya., Zhang L., Wang J. Topological Anderson insulator phenomena. Phys. Rev. B, 2011, 84, P. 035110. 